\title{
Self consistent modelling of the projection factor for interferometric distance determination
}

\author{
N. Nardetto ${ }^{1}$, A. Fokin ${ }^{1,2,5}$, D. Mourard ${ }^{1}$, Ph. Mathias ${ }^{1}$, P. Kervella ${ }^{3}$, and D. Bersier ${ }^{4}$ \\ 1 Observatoire de la Côte d'Azur, Dpt. Gemini, UMR 6203, 06130 Grasse, France \\ e-mail: Nicolas. Nardetto@obs-azur.fr \\ 2 Institute of Astronomy of the Russian Academy of Sciences, 48 Pjatnitskaya Str., Moscow 109017, Russia \\ 3 Observatoire de Paris-Meudon, LESIA, UMR 8109, 5 place Jules Janssen, 92195 Meudon Cedex, France \\ ${ }^{4}$ Space Telescope Science Institute, 3700 San Martin Drive, Baltimore, MD 21218, USA \\ 5 Isaak Newton Institute, Moscow Branch
}

Received 4 June 2004 / Accepted 28 July 2004

\begin{abstract}
The distance of galactic Cepheids can be derived through the interferometric Baade-Wesselink method. The interferometric measurements lead to angular diameter estimations over the whole pulsation period, while the stellar radius variations can be deduced from the integration of the pulsation velocity. The latter is linked to the observational velocity deduced from line profiles by the so-called projection factor $p$. The knowledge of $p$ is currently an important limiting factor for this method of distance determination. A self-consistent and time-dependent model of the star $\delta$ Cep is computed in order to study the dynamical structure of its atmosphere together with the induced line profile. Different kinds of radial and pulsation velocities are then derived. In particular, we compile a suitable average value for the projection factor related to different observational techniques, such as spectrometry, and spectral-line or wide-band interferometry. We show that the impact on the average projection factor and consequently on the final distance deduced from this method is of the order of $6 \%$. We also study the impact of a constant or variable $p$-factor on the Cepheid distance determination. We conclude on this last point that if the average value of the projection factor is correct, then the influence of the time dependence is not significant as the error in the final distance is of the order of $0.2 \%$.
\end{abstract}

Key words. stars: atmospheres - stars: distances - stars: oscillations - stars: variables: Cepheids

\section{Introduction}

The period-luminosity $(\mathrm{P}-\mathrm{L})$ relation of the Cepheids is the basis of the extragalactic distance scale, but its calibration is still uncertain at a $\Delta M= \pm 0.10 \mathrm{mag}$ level. In order to calibrate this relation, two procedures have been recently considered, both based on the Baade-Wesselink method (hereafter $\mathrm{BW}$ ), with distances deduced from the ratio of radius to angular variations.

The first method is the near-infrared surface brightness method introduced by Welch (1994), and later by Fouqué \& Gieren (1997). The angular diameter variation is photometrically inferred from calibrations of the $V$ light and $(V-K)$ color curves, and compared to the radius variation obtained spectroscopically. In the second method, called the interferometric version of the Baade-Wesselink method (hereafter IBW), the angular diameter variation is directly measured through the latest generation of long-baseline interferometers in the visible and in the IR, and then again compared to radius variations in order to derive distances (Kervella et al. 2004a; Lane et al. 2002) and then calibrate the $\mathrm{P}-\mathrm{L}$ relation (Kervella et al. 2004b).
Both methods are in perfect agreement on the angular diameter, with a discrepancy of less than $1.5 \%$ (Kervella et al. $2004 c$ ). However, a difficulty remains in the derivation of the radius variation. The radius displacement is obtained through the integration of the pulsation velocity curve, hereafter called $v_{\text {puls }}$. But when one measures radial velocities from line profiles, hereafter called $v_{\text {rad }}$, they include the integration in two directions over the surface, through limb-darkening, and over the radius, through velocity gradients in line forming regions. Moreover, both the limb-darkening and velocity gradients depend on the pulsation phase, as already pointed out by Marengo et al. (2003). Therefore, the knowledge of the projection factor, defined as $v_{\text {puls }}=p * v_{\text {rad }}$, is of crucial importance for deriving a correct estimate of the radius variation curves from the integration of the pulsation velocity curve.

\subsection{Previous work}

The problem of the projection factor has been first studied by Eddington (1926), Carroll (1928) and Getting (1935). These authors consider both effects of limb-darkening and 
atmospheric expansion at constant velocity on the line profile. These studies led to a $p$-value of $\frac{24}{17}=1.41$, which was used for several decades in the Baade-Wesselink method.

Later, Van Hoof \& Deurinck (1952) showed that when the natural width of the line is much smaller than the shift induced by the Doppler effect, the resulting profile must be distorted, and the $p$-factor can be measured from the convolution of the static line profile with this distortion function. Parsons (1972), using a model atmosphere with uniform expansion, numerically determined $p$-values between 1.31 and 1.34 depending on the width of the line.

Karp $(1973,1975)$ introduced a velocity gradient within the line forming regions and computed the emerging flux for both weak and strong lines. Weak lines, appear asymmetrical similar to the ones obtained by Van Hoof \& Deurinck (1952), while the distortion for the stronger lines is mainly due to the velocity gradient within the atmosphere. Albrow \& Cottrell (1994) determine values for $p$ larger by $10 \%$ than those obtained by Parsons (1972), a difference interpreted as due to the use of a different limb-darkening law. Indeed, the $p$-factor depends on many parameters, such as the wavelength ( $p$ is larger in the infrared, Sasselov \& Lester 1990), or the effective temperature of the star (Hindsley \& Bell 1986; Montañés Rodriguez \& Jeffery 2001).

From an observational point of view, Burki et al. (1982) determined $p=1.36$ from the measure of the centroid of the correlation profile, a value which has been widely used in spectroscopy.

Finally, since $p$ is determined both through geometrical effects and atmospheric dynamics, which change during the pulsation cycle, it should itself vary with the pulsation phase. In particular, Sabbey et al. (1995) showed that this effect on $p$ can increase the BW radius by about $6 \%$.

\subsection{This work}

We apply for $\delta$ Cep a nonlinear self-consistent hydrodynamical model (Fokin 1990). In addition, radiative transfer is considered in the outer layers to produce a realistic atmosphere model. The derived quantities have been found to be in good agreement with observations for different classes of pulsators such as RR Lyrae (Fokin \& Gillet 1997), RV Tauri (Fokin 2001), postAGB (Jeannin et al. 1997), BL Herculis (Fokin \& Gillet 1994) and more recently $\beta$ Cephei stars (Fokin et al. 2004). In particular, this model has already been used in the case of $\delta$ Cep (Fokin et al. 1996). Our model has some limitations (no convection, no adaptive grid), but is able to reproduce the main observational characteristics such as the presence of shocks or the correct shape and amplitude of the velocity curve. Thus we are confident that our model is valid for our study, and that the results are consistent.

The influence of the projection factor on the distance determination of Cepheids can be safely studied in the context of the IBW method. The main objectives of this paper are, firstly, to have an idea of the best value of the $p$-factor for interferometric observations, and to compare it with the generally used value of $p=1.36$, and secondly, to quantify the impact of a constant or time-dependent projection factor on the distance determination of the star.

The paper is organized as follows. In Sect. 2 we describe our model of the prototype of the Cepheids, $\delta$ Cep, constrained from observational parameters referenced in the literature. In Sect. 3, we define the radial and pulsation velocities considered in the following. Section 4 deals essentially with the study of the projection factor and Sect. 5 concerns the impact of the choice of a time-varying $p$-factor on the distance determination. Finally, Sect. 6 presents the conclusions of this work.

\section{The model of $\delta$ Cep}

The model needs only 4 input parameters: the luminosity $(L)$, the effective temperature $\left(T_{\text {eff }}\right)$, the mass $(M)$ and the chemical composition $(X$ and $Y)$. The model is run until it reaches its limit cycle (for $\delta$ Cep this is the fundamental mode). Radiative transfer in the line is then solved in the frame of this hydrodynamical model to provide line profiles (Fokin 1991). For the present study, which is a first step, we have arbitrarily considered the metallic line Fe I 6003.012 A. Therefore, we can compare the velocity in a given mass zone $\left(v_{\text {puls }}\right)$ with the velocity measured from the synthetic line profile $\left(v_{\mathrm{rad}}\right)$. The latter was determined by two methods: measuring the velocity associated with the pixel at the minimum of the line profile (hereafter called profile minimum), and the Gaussian method in which we fit the whole profile with a Gaussian function. Note that theoretical variations follow the usual convention in which the pulsation phase $\phi=0$ corresponds to maximum luminosity.

Since the main stellar quantities of $\delta$ Cep (HD 213306) are still uncertain, we tried several sets of luminosity $L$, effective temperature $T_{\text {eff }}$ and mass $M$ in order to get suitable observational quantities such as the pulsation period, the average radius of the star, bolometric and radial velocity curves, and line profiles. This leads to the following set for the 106-zone model: $M=4.8 M_{\odot}, L=1995 L_{\odot}, T_{\text {eff }}=5877 \mathrm{~K}$. This latter is in agreement with the one measured by Fernley et al. (1989). Mass and luminosity are related through the M-L relation of Chiosi et al. (1993):

$\log \frac{L}{L_{\odot}}=-0.015+3.14 Y-10.0 Z+3.502 \log \frac{M}{M_{\odot}}+0.25$

where $Y=0.28$ and $Z=0.02$ correspond to typical Pop. I chemical composition. The inner boundary has been fixed at about $T=1.0 \times 10^{6} \mathrm{~K}$, corresponding to about $16 \%$ of the photospheric radius, so the model envelope with the atmosphere contains about $7 \%$ of the stellar mass. The atmosphere itself contains about $1.0 \times 10^{-7}$ of the total stellar mass. In the hydrodynamical model we used the OPAL92 opacity table. Note that in the following line transfer calculation for each chosen phase we used the snapshots of the pulsating atmosphere given by the nonlinear model. In addition, we used the relevant frequencydependent atomic opacities both in the continuum and in the line.

We started the hydrodynamical calculations with an initial velocity profile with a value of $25 \mathrm{~km} \mathrm{~s}^{-1}$ at the surface. At the limit cycle the pulsation period is 5.419 days, very close $(1 \%)$ to the observational value deduced by 


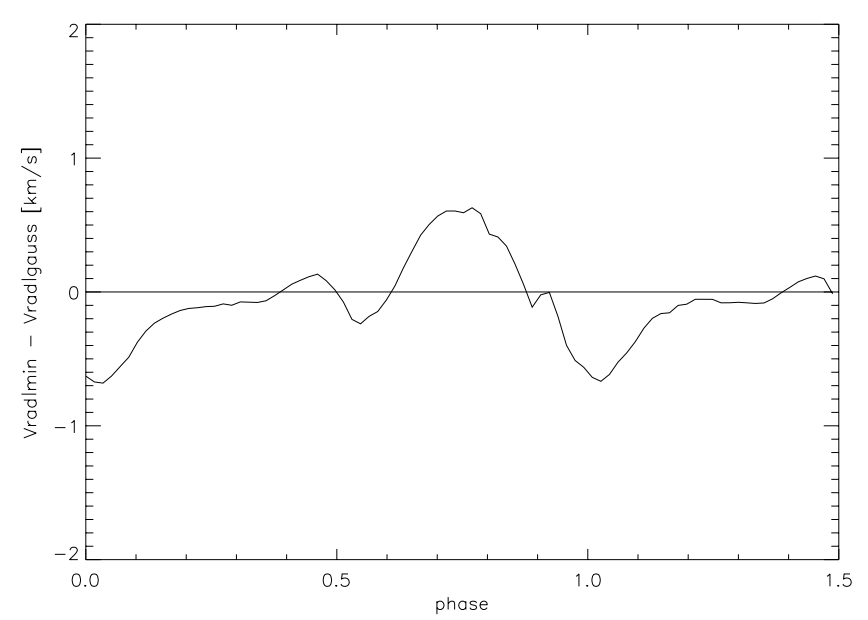

Fig. 1. Difference between the theoretical radial velocity curves measured by the profile minimum method $\left(v_{\text {rad } \mid \min }\right)$ and the Gaussian fitting method $\left(v_{\text {radgauss }}\right)$. The small difference induces a bias in the determination of the $p$-factor. The horizontal line is the zero velocity in the stellar rest frame.

Szabados et al. (1980). Bolometric and radial velocity amplitudes are respectively $\Delta m_{\text {bol }}=0.85 \mathrm{mag}$ and $\Delta V=35 \mathrm{~km} \mathrm{~s}^{-1}$. The relative radius amplitude at the surface is $\Delta R / R=10 \%$. The mean photospheric radius is about $\bar{R}=43.5 R_{\odot}$, in agreement with interferometric and parallax measurements obtained by Mourard et al. (1997), Nordgren et al. (2000) and Benedict et al. (2002).

We then generated a series of snapshots of the atmospheric structure (about 60 per pulsation period) and after the line profile computation we deduced the radial velocity variations. For all phases we assume the same microturbulence velocity of $1 \mathrm{~km} \mathrm{~s}^{-1}$, and we neglect the rotation $\left(v \sin i \sim 5 \mathrm{~km} \mathrm{~s}^{-1}\right.$, Breitfellner \& Gillet 1993).

\section{Velocities}

To study the projection factor, we now define different radial and pulsational velocities.

\subsection{The radial velocity}

Theoretical line profiles deduced from the $\delta$ Cep model are used to determine apparent radial velocities considering either the minimum of the profile or the Gaussian fit. The maximum velocity difference between these two methods reaches about $0.7 \mathrm{~km} \mathrm{~s}^{-1}$ during extrema phases $(\phi=0.7-0.8$ and $\phi=0.9-0.1$ ), see Fig. 1. We will show later that such a difference is not negligible for the projection factor determination.

\subsection{The pulsation velocity}

The projection factor may have different definitions depending on the pulsating layer considered. From a spectroscopic point of view, one considers the gas velocity associated to the optical barycenter of the line forming region. However, the instruments, spectrograph and interferometer, do not probe the same layers of the star. For instance, with the IBW method, the layers that are seen by the interferometer depend on the spectral resolution. Indeed, a wide spectral band will rather probe the continuum (photospheric) region. Conversely, in a specific line, the visibility function is the Fourier transform of the image of the star in the considered line. Thus different cases, corresponding to each type of observation, have to be considered.

Firstly, for spectroscopic observations the gas velocity is that of the line-forming layers. However this region may represent an appreciable fraction of the height of the atmosphere. Sabbey et al. (1995) determined the layer corresponding to the optical center of gravity of the line from contribution functions. In our case, we consider the standard definition in which the line core is formed at an optical depth of $\tau=2 / 3$. Hence, we use the definition:

$v_{\operatorname{puls}_{(\mathrm{s})}}=v\left(\tau_{l}=\frac{2}{3}\right)$

where $\tau_{l}$ is the optical depth at the center of the line and "(s)" means "Spectroscopy".

Secondly, for interferometric observations in one particular line, it is better to consider the velocity of optical layers corresponding to an optical depth of $\tau_{l}=2 / 3$. It is not the gas velocity that is considered here but the velocity of the optical layer deduced from the pulsation model, defined by:

$v_{\text {puls }_{(\mathrm{il})}}=\frac{\partial R\left(\tau_{l}=2 / 3\right)}{\partial \phi}$

where "(il)" is for "Interferometry in one Line".

Similarly, for interferometric observations in a wide band, the most appropriate pulsation velocity is the one associated to the photospheric layer that corresponds, by definition, to $\tau=$ $2 / 3$ in the continuum:

$v_{\text {puls }_{(\mathrm{ic})}}=\frac{\partial R\left(\tau_{\mathrm{c}}=2 / 3\right)}{\partial \phi}$

where "(ic)" is for "Interferometry in the Continuum".

Note that we consider here the continuum next to the line. Figure 2 represents the different pulsation velocities defined above. These three pulsation velocity curves are different by a maximum of $5 \%$ during the extrema phases, because the atmosphere is not co-moving. The asymmetry in the profile is maximum during the phases of extrema of the radial velocity curve, thus there should be a large velocity gradient between the different layers.

\section{The projection factor}

\subsection{Combination of radial and pulsation velocities}

It is now possible to combine the radial velocities (two cases) with the pulsation velocities (three cases) to derive the projection factor. Figures $3 \mathrm{a}-\mathrm{c}$ shows the three pulsation velocities together with the radial velocity using the Gaussian method. Note that the estimators of radial and pulsation velocities, in (s) and (il) cases, are supposed to probe the same part of the star, the line forming region. In other words the two curves should cancel at the same phase respectively in Figs. $3 \mathrm{a}$ and $3 \mathrm{~b}$, which 


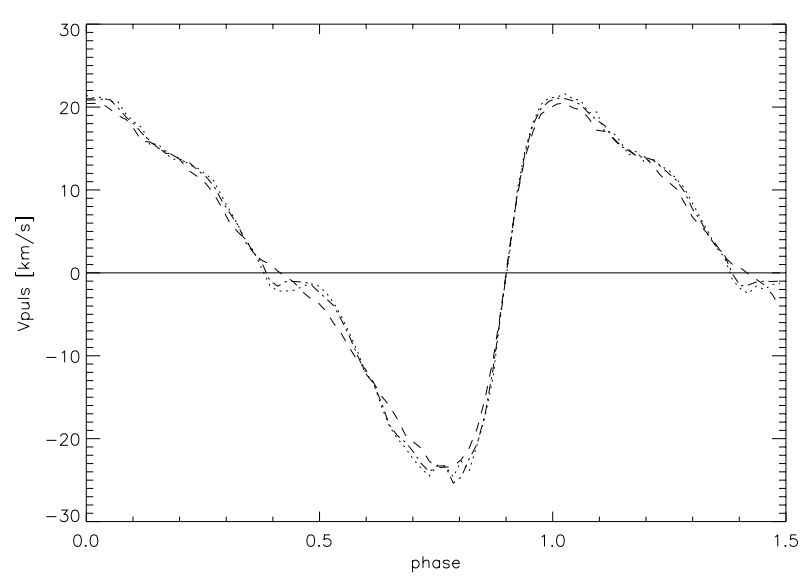

Fig. 2. Pulsation velocities vs. phase. The dashed curve shows the velocity of the photospheric layer ( $\tau=2 / 3$ in the continuum), the dotdashed curve the velocity of the layer corresponding to $\tau=2 / 3$ in the spectral center of the line and the dotted curve the gas velocity corresponding to $\tau=2 / 3$ in the line. The horizontal line is the zero velocity in the stellar rest frame.

is actually the case with a good precision $(\phi \sim 0.4)$. This is an indication that our estimator of the optical barycenter $\tau=2 / 3$ is correct. The result should have been the same considering the profile minimum as the velocity curve cancels at the same phase (see $\phi=0.4$ in Fig. 1). However, we note in Fig. 3c that the zero point of the photospheric velocity is at a slightly later phase. This is the result of asynchronous motions in the atmosphere.

All these curves, with their amplitude and shape, will have an impact on the projection factor and its variation over the pulsation. In the following section we compute a suitable average value of the projection factor for each case, considering two estimators which are not simply the average of the ratio of pulsation to radial velocities. In Sect. 5, we consider more specifically the time dependence of the projection factor.

\subsection{Two estimators of $p$}

To determine a constant projection factor, we cannot simply consider the mean value of the ratio of the pulsation to radial velocities. Due to the non-comoving character of the atmospheric motions, this would lead to a ratio of physical quantities close to zero $(\phi \sim 0.4)$ but not exactly at the same phase, whatever the case considered in Fig. 3. Consequently, two more suitable tests were used to estimate a constant value of $p$. The first consists in applying a classical $\chi^{2}$ minimization algorithm between the quantity $v_{\text {rad }} \cdot p_{\text {const }}$ and the considered pulsation velocity (hereafter estimator 1 ):

$\chi^{2}=\sum_{i} \frac{\left(v_{\mathrm{rad}}\left(\phi_{i}\right) \cdot p_{\mathrm{const}}-v_{\text {puls }}\left(\phi_{i}\right)\right)^{2}}{\sigma_{\text {puls }}\left(\phi_{i}\right)^{2}}$

where $\sigma_{\text {puls }}\left(\phi_{i}\right)$ is the statistical error in the pulsation velocity, arbitrarily fixed to a reasonable value of $1 \mathrm{~km} \mathrm{~s}^{-1}$, in order to evaluate the corresponding error on $p$. The phases $\phi_{i}$, in this case, are sampled following the snapshots of the model. Here,
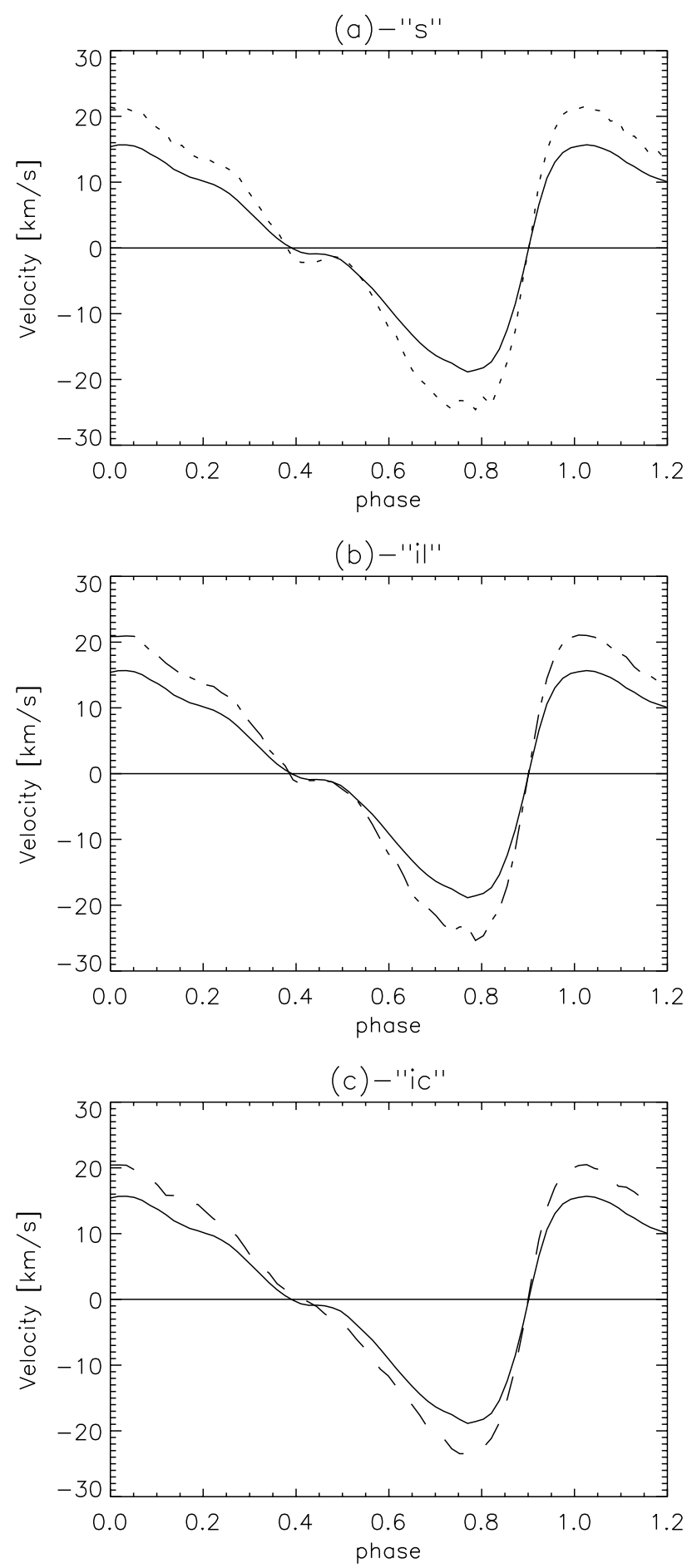

Fig. 3. Radial velocity curve deduced from the theoretical line profiles by the Gaussian method together with a) the gas velocity corresponding to $\tau=2 / 3$ in the line forming region according to Eq. (1), b) the $\tau=2 / 3$ "optical layer" velocity according to Eq. (2), c) the velocity of the photospheric layer ( $\tau=2 / 3$ in the continuum, see Eq. (3)).

$v_{\text {rad }}$ is the radial velocity deduced from either the profile minimum or the Gaussian fit, and $v_{\text {puls }}$ is related to Eqs. (1)-(3).

The second estimator of the $p$-factor is directly based on the radius variation of the star, obtained either by integration of the radial velocity or directly by the position of the layer as 
Table 1. Optimal constant values for the $p$-factor for different cases of interest. $v_{\text {radgauss }}$ and $v_{\text {rad|min }}$ are the radial velocity deduced from theoretical line profiles using the Gaussian and minimum method respectively. Estimator (1) and (2) of the constant projection factor correspond to Eqs. (4) and (5) respectively. In each case the pulsational velocity $v_{\text {puls }}$ and radius $\Delta R_{\text {puls }}$ used are indicated.

\begin{tabular}{lcc}
\hline \hline & Estimator 1 & Estimator 2 \\
\hline & $v_{\text {pul }_{(\mathrm{s})}}=v\left(\tau_{l}=2 / 3\right)$ & $\Delta R_{\text {pul }_{(\mathrm{s})}}=\int v\left(\tau_{l}=2 / 3\right)$ \\
$v_{\text {rad|gauss }}$ & $1.35 \pm 0.01$ & $1.32 \pm 0.01$ \\
$v_{\text {rad|min }}$ & $1.31 \pm 0.01$ & $1.30 \pm 0.01$ \\
\hline & $v_{\text {pul }_{(\mathrm{il})}}=\frac{\partial R\left(\tau_{l}=2 / 3\right)}{\partial \phi}$ & $\Delta R_{\text {puls }_{(\mathrm{il})}}=\Delta R\left(\tau_{l}=2 / 3\right)$ \\
$v_{\text {rad|gauss }}$ & $1.33 \pm 0.01$ & $1.32 \pm 0.01$ \\
$v_{\text {rad|min }}$ & $1.30 \pm 0.01$ & $1.29 \pm 0.01$ \\
\hline & $v_{\text {puls }_{(\mathrm{ic})}}=\frac{\partial R\left(\tau_{\mathrm{c}}=2 / 3\right)}{\partial \phi}$ & $\Delta R_{\text {puls }_{(\text {(i) }}}=\Delta R\left(\tau_{\mathrm{c}}=2 / 3\right)$ \\
$v_{\text {rad|gauss }}$ & $1.28 \pm 0.01$ & $1.27 \pm 0.01$ \\
$v_{\text {rad|min }}$ & $1.24 \pm 0.01$ & $1.24 \pm 0.01$ \\
\hline
\end{tabular}

provided by the radius of the mass zone involved. Hence, the quantity defined by $\int v_{\text {rad }} \cdot p_{\text {const }}$ is compared with the pulsating radius (hereafter estimator 2 ):

$\chi^{2}=\sum_{i} \frac{\left(\int v_{\mathrm{rad}}\left(\phi_{i}\right) \cdot p_{\text {const }}-\Delta R_{\text {puls }}\left(\phi_{i}\right)\right)^{2}}{\sigma_{\text {puls }}\left(\phi_{i}\right)^{2}}$

where $\sigma_{\text {puls }}\left(\phi_{i}\right)$ is the statistical error in the pulsation radius, fixed to $0.1 R_{\odot}$ to obtain the same uncertainty in the $p$-factor for both estimators. The quantity $v_{\text {rad }}$ is the same as in estimator 1 . The radius variation $\Delta R_{\text {puls }}$ may be either:

$\Delta R_{\operatorname{puls}_{(\mathrm{s})}}=\int v\left(\tau_{l}=2 / 3\right)$

or

$\Delta R_{\text {puls }_{(\mathrm{il})}}=\Delta R\left(\tau_{l}=2 / 3\right)$

or

$\Delta R_{\text {puls }_{(\mathrm{ic})}}=\Delta R\left(\tau_{\mathrm{c}}=2 / 3\right)$,

with each case corresponding theoretically to the integration of Eqs. (1)-(3). However, note that for Eqs. (7) and (8) the radius variations are deduced directly from the model. An integration algorithm was used to derive Eq. (6). We also define $R_{\text {puls }}=$ $\overline{R_{\text {puls }}}+\Delta R_{\text {puls }}$ for each case.

\subsection{Results and discussion}

Table 1 lists the computation results for the twelve cases considered, leading to the following conclusions.

Firstly, the $p$-factors obtained considering the two estimators differ by $2 \%$ in extreme cases. This is expected for two reasons. On the one hand, the two minimized quantities are different, so it is expected that the associated $p$-factor values will also be different. On the other hand, when the radius is fitted, the estimator may be less sensitive to velocity variation shapes.
Secondly, these results indicate a systematic shift of $0.02-0.04$ (or 3\%) in $p$-values between the radial velocities associated with the Gaussian and the profile minimum methods. This is logically linked to the systematic difference in velocity curves, as shown in Fig. 1. Therefore it is important to choose the $p$-factor value that corresponds to the method that was used to estimate the projected velocity. In addition, it is best to use the method that is least sensitive to velocity gradients and marginal effects, in order to obtain a value for the $p$-factor that is generally applicable. That is why in the following discussions, we consider only the radial velocity deduced from the Gaussian method.

Thirdly, the difference between the pulsation layers considered should be related to the different observational techniques, as we pointed out in Sect.3. For spectroscopic measurements of the gas velocity within the line, the recommended value is $p=1.35$, which is close to the classical value of $p=1.36$ (Burki et al. 1982). In this case, one should preferably consider the first estimator since one has to deal with the pulsation velocity of the gas to account for the atmosphere dynamics. Conversely, for interferometric observations in a "photospheric" line, the best value is $p=1.32$, and one should consider the second estimator (this result will be confirmed in the next section). For broadband interferometric observations, one should use a lower value of $p=1.27$. These results indicate that an error of $6 \%$ can be made if one takes the usual value of $p=1.36$ regardless of the observational method used.

Finally, an initial error of $1 \mathrm{~km} \mathrm{~s}^{-1}$ in the pulsation velocity, or $0.1 R_{\odot}$ in the pulsation radius, leads to a final statistical error in the $p$-factor of about 0.01 for both estimators.

\section{The effect of a constant projection factor on distance determination}

The IBW method combines interferometric and spectrometric observations to deduce the distance of the star (see Kervella et al. 2004a). In the previous section we have obtained different average values for $p$, considering different kinds of velocities and estimators. We now study the influence of the time-dependence of $p$ on distance determination. Since the definition of $p$ involves phase-dependent factors, $p$ itself should be time-dependent. This is illustrated in Fig. 4 which shows the quantity $v_{\text {puls }}-p_{\text {const }} * v_{\text {rad }}$. As it has already been pointed out in Sect. 4.2, plotting the $p$-factor against the phase is misleading as the ratio of pulsation to radial velocities is not representative when these quantities are close to zero $(\phi \sim 0.4)$. Moreover, in the framework of the IBW method, the quantity of interest is the pulsation velocity rather than the projection factor itself.

On the one hand, we simulate angular diameters $\theta_{\mathrm{obs}}$, fixing arbitrarily the distance of the star $(d=275 \mathrm{pc})$ and using the radius variations provided by the pulsation model:

$\theta_{\text {obs }}\left(\phi_{i}\right)=9.305\left(\frac{R_{\text {puls }}\left(\phi_{i}\right)}{275}\right)[$ mas $]$

where $R_{\text {puls }}$ (in $R_{\odot}$ ) is one of the three quantities:

$R_{\text {puls }_{(\mathrm{s})}}=\int v\left(\tau_{l}=2 / 3\right)$ 


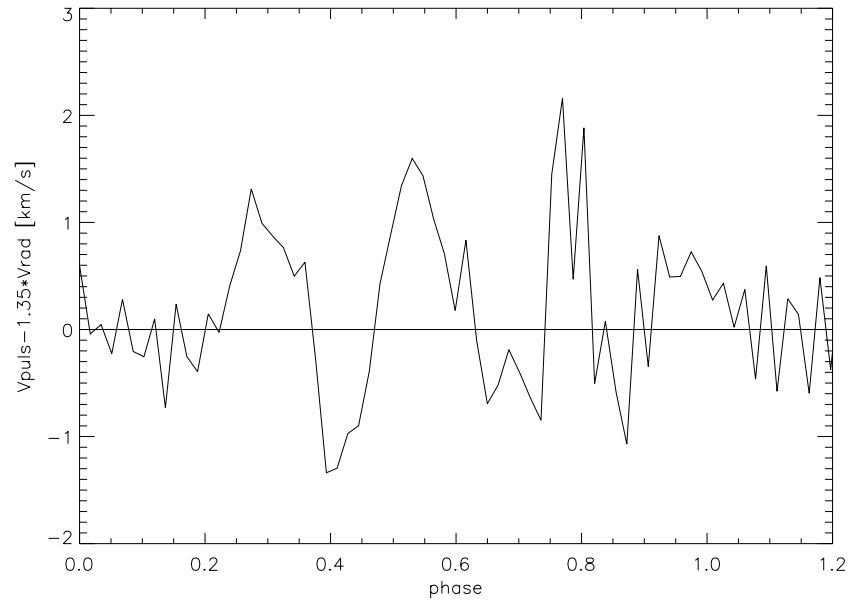

Fig. 4. The quantity $v_{\text {puls }}-1.35 * v_{\text {rad }}$ versus the phase in the case of Fig. 3a: $v_{\text {puls }}$ is the gas velocity corresponding to $\tau=2 / 3$ in the line formation region according to Eq. (1), and $v_{\text {rad }}$ is the radial velocity curve deduced from the theoretical line profiles by the Gaussian method. $p=1.35$ is the optimum value obtained from the estimator 1 , as described in Sect. 4.2

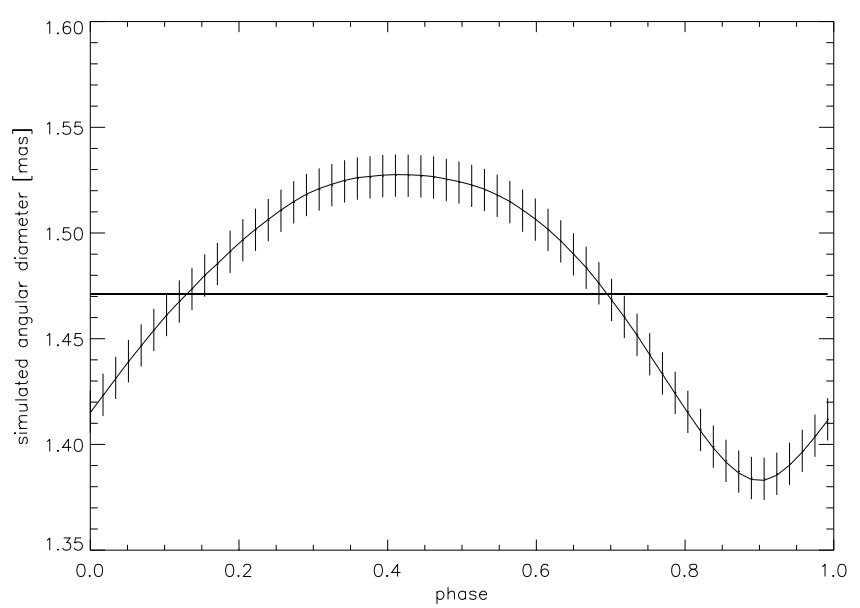

Fig. 5. Simulated angular diameter points deduced from Eq. (9) with $R_{\text {puls }_{\text {(ic) }}}=R\left(\tau_{\mathrm{c}}=2 / 3\right)$. Each point is shown with its arbitrary theoretical error bar of 0.01 mas. This curve simulates interferometric observations used in the IBW method.

or

$R_{\text {puls }_{(\mathrm{il})}}=R\left(\tau_{l}=2 / 3\right)$

or

$R_{\text {puls }_{(\mathrm{ic})}}=R\left(\tau_{\mathrm{c}}=2 / 3\right)$,

as provided by the integration of Eqs. (1)-(3). The phases $\phi_{i}$ are sampled from the snapshots of the model. Figure 5 shows the simulated angular diameter curve considering $R_{\text {puls }_{(\mathrm{ic})}}=R\left(\tau_{\mathrm{c}}=\right.$ 2/3).

On the other hand, the IBW method is used as follows. Firstly, a radial velocity curve is derived from the synthetic spectra considering both the Gaussian fit and the minimum profile methods. Then, a constant value for the $p$-factor is chosen corresponding to one of the twelve cases of Table 1. Finally,
Table 2. Distance results corresponding to the mean $p$-factor results of Table 1. The different expressions of the radius refer to Eqs. (10)-(12) respectively and correspond to the quantity used in the Eq. (9) of the simulated angular diameters.

\begin{tabular}{ccc}
\hline \hline & Estimator 1 & Estimator 2 \\
\hline & \multicolumn{2}{c}{$R_{\text {puls }}=\int v\left(\tau_{l}=2 / 3\right)$} \\
$v_{\text {rad|gauss }}$ & $279.6 \pm 7.2$ & $274.6 \pm 7.2$ \\
$v_{\text {rad|min }}$ & $278.2 \pm 7.2$ & $274.8 \pm 7.2$ \\
\hline \multicolumn{3}{c}{$R_{\text {puls }}=R\left(\tau_{l}=2 / 3\right)$} \\
$v_{\text {rad|gauss }}$ & $278.2 \pm 7.2$ & $274.6 \pm 7.2$ \\
$v_{\text {rad|min }}$ & $276.9 \pm 7.2$ & $274.7 \pm 7.2$ \\
\hline \multicolumn{3}{c}{$R_{\text {puls }}=R\left(\tau_{\mathrm{c}}=2 / 3\right)$} \\
$v_{\text {rad|gauss }}$ & $276.0 \pm 7.2$ & $274.9 \pm 7.2$ \\
$v_{\text {rad|min }}$ & $274.6 \pm 7.2$ & $274.8 \pm 7.2$ \\
\hline
\end{tabular}

the integration of the pulsation velocity deduced from the radial velocity and the projection factor leads to an estimation of the radius variation of the star. This leads to an angular variation curve:

$\theta_{\text {model }}\left(\phi_{i}\right)=\bar{\theta}+9.305\left(\frac{\Delta R\left(\phi_{i}\right)}{d}\right)[$ mas $]$,

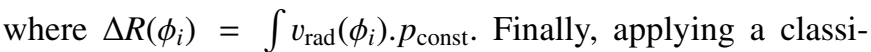
cal $\chi^{2}$ minimization algorithm, we fit both the average angular diameter $\bar{\theta}$ and the distance $d$ to the star. The minimized quantity is:

$\chi^{2}=\sum_{i} \frac{\left(\theta_{\mathrm{obs}}\left(\phi_{i}\right)-\theta_{\text {model }}\left(\phi_{i}\right)\right)^{2}}{\sigma_{\mathrm{obs}}\left(\phi_{i}\right)^{2}}$.

The values for $\sigma_{\mathrm{obs}}\left(\phi_{i}\right)$ are arbitrarily fixed to 0.01 mas which is a realistic value considering the measurement precision achieved recently by long-baseline interferometers (see Fig. 5).

Table 2 gives the computed distances using the $p$-factors shown in Table 1 . The mean angular diameters obtained correspond to the anticipated values of $\overline{\theta_{\mathrm{obs}}}=1.471 \mathrm{mas}$ for the (ic) case and $\overline{\theta_{\mathrm{obs}}}=1.476$ for (s) and (il) cases. The statistical errors obtained are around 0.001 mas.

Since $p$ is constant, we have $\Delta R \sim \Delta R_{\text {puls }}$, and any departure from the predefined distance $(275 \mathrm{pc})$ is the result of the time-dependence of the projection factor or the choice of the estimator: there is no model effect. It appears that the computed and reference distances are closer for estimator 2 . Thus, estimator 2 provides projection factors less biased than those provided by estimator 1 in the frame of the IBW method.

An important conclusion is that for the best $p$-factor value, the systematic error in the derived distance does not exceed $0.2 \%$, independent of the radial and pulsation velocities considered. This important result indicates that a time-dependent $p$-factor is not required at the moment since the final error of $0.2 \%$ is well below our best estimation of recent distance determination.

Finally, note that the initial uncertainty of 0.01 mas in theoretical angular diameters leads to a final statistical error in the distance of $7.2 \mathrm{pc}$. 


\section{Conclusion}

A self-consistent nonlinear model for $\delta$ Cephei was generated reproducing the main observational features of this star.

On the basis of this model we studied the effect of the projection factor which links radial and pulsation velocity on the IBW method for distance determination. Two methods were considered for deriving the radial velocity curve: a Gaussian fit and the profile-minimum method. Similarly, three pulsation velocities were defined corresponding to different regions of the stellar atmosphere: two concern the line forming region, while the third corresponds to the photosphere. These three pulsation velocities are linked to different observational techniques such as spectrometry and wide-band or spectral-line interferometry. An important result of this study is the very weak influence of the time-dependence of the $p$-factor on distance determination. The choice of a constant $p$-factor instead of one that is timedependent gives a systematic error in the final distance of the order of $0.2 \%$, which is below the best estimations of current distance determination. More important, the projection factor should be chosen depending on the observational techniques used. For spectroscopic observations, if we use the Gaussian method to derive the radial velocity, we propose $p=1.35$. For wide-band interferometry, the best value is $p=1.27$, and for interferometric observations in a specific (metal) line it is $p=1.32$. Note that this latter value has been determined for a given line: considering lines formed in other atmospheric regions should lead to different values. An extensive study of this dependence, outside the scope of the present paper, is currently in progress.

Note also that these values have been determined for $\delta$ Cep. The generalization of our results to other classical Cepheids will require the study of a larger sample of stars. The AMBER instrument (Petrov et al. 2000) will also permit observations in one particular line with a good resolution ("Interferometry in one Line"). Cepheids are bright sources and observations in an absorption line of their atmospheres appears feasible in terms of signal to noise ratio of as long as one can use large telescopes and adaptive optics. It will be then possible to compare the same layer of the star with interferometry and spectrometry.

Acknowledgements. We thank $\mathrm{Ph}$. Berio for having provided the $\chi^{2}$ minimization algorithm and for useful discussions. A. Fokin acknowledges the Observatoire de la Côte d'Azur for financial support.

\section{References}

Albrow, M. D., \& Cottrell, P. L. 1994, MNRAS, 267, 584

Benedict, G. F., McArthur, B. E., Fredrick, L. W., et al. 2002, AJ, 124, 1695

Breitfellner, M. G., \& Gillet, D. 1993, A\&A, 277, 524B

Burki, G., Mayor, M., Benz, W. 1982, A\&A, 109, 258

Caroll, M. A. 1928, MNRAS, 88, 548

Chiosi, C., Wood, P. R., \& Capitanio, N. 1993, ApJS, 86, 541

Eddington, A. S. 1926, The Internal Constitution of the Stars (Cambridge University Press), 185

Fernley, J. A., Skillen, I., \& Jameson, R. F. 1989, MNRAS, 237, 947

Fokin, A. B. 1990, Ap\&SS, 164, 95

Fokin, A. B. 1991, MNRAS, 250, 258

Fokin, A. B. 2001, in Stellar pulsation - nonlinear studies ASSL series, ed. M. Takeuti, \& D. D. Sasselov, 257, 103

Fokin, A. B., \& Gillet, D. 1994, A\&A, 290, 875

Fokin, A. B., \& Gillet D. 1997, A\&A, 325, 1013

Fokin, A. B., Gillet, D., \& Breitfellner, M. G. 1996, A\&A, 307, 503

Fokin, A. B., Mathias, Ph., Chapellier, E., Gillet, D., \& Nardetto, N. 2004, A\&A, 426, 687

Fouqué, P., \& Gieren, W. P. 1997, A\&A, 320, 799

Getting, I. A. 1935, MNRAS, 95, 141

Hindsley, R., \& Bell, R. A. 1986, PASP Conf. Ser., 98, 881

Jeannin L., Fokin A. B., Gillet D., \& Baraffe I. 1997, A\&A, 326, 203

Karp, A. H. 1973, ApJ, 180, 895

Karp, A. H. 1975, ApJ, 201, 641

Kervella, P., Nardetto, N., Bersier, D., et al. 2004a, A\&A, 416, 941

Kervella, P., Bersier, D., Mourard D., Nardetto, N., \& Coudé Du Foresto, V. 2004b, A\&A, 423, 327

Kervella, P., Fouqué, P., Storm, J., et al. 2004, ApJ, 604, L113

Lane, B. F., Creech-Eakman, M., \& Nordgren, T. E. 2002, ApJ, 573, 330

Parsons, S. B. 1972, ApJ, 174, 57

Petrov, R., Malbet, F., Richichi, A., et al. 2000, Proc. SPIE, 4006, 68

Marengo, M., Karovska, M., Sasselov, D. D., et al. 2003, ApJ, 589, 975

Montañés Rodriguez, P., \& Jeffery, C. S. 2001, A\&A, 375, 411

Mourard, D., Bonneau, D., Koechlin, L., et al. 1997, A\&A, 317,789

Nordgren, T. E., Armstrong, J. T., Germain, M. E., et al. 2000, ApJ, 543, 972

Sabbey, C. N., Sasselov, D. D., Fieldus, M. S., et al. 1995, ApJ, 446, 250

Sasselov, D. D., \& Lester, J. B. 1990, ApJ, 362, 333

Szabados L. 1980, Comm. Konkoly Obs. Hungary., 76, 1

Van Hoof, A., \& Deurinck, R. 1952, ApJ, 115, 166

Welch, D. L. 1994, AJ, 108, 1421 